\title{
Inseguridad subjetiva y representaciones sociales de la delincuencia*
}

\section{Subjective Insecurity and Social Representations of Delinquency}

Recepción: 28 Mayo 2015 | Aprobación: 27 Junio 2017

\author{
Ainara Arnoso Martínez \\ Universidad del País Vasco, España \\ ORCID: http://orcid.org/0000-0001-5472-9508 \\ Laura Vozmediano SAnZ \\ Universidad del País Vasco, España \\ Cristina Martínez de Taboada Kutz \\ Universidad del País Vasco, España
}

a Autor de correspondencia. Correo electrónico: ainara.arnoso@ehu.eus

Para citar este artículo: Arnoso Martínez, A., Vozmediano Sanz, L., \& Martínez de Taboada Kutz, C. (2018). Inseguridad subjetiva y representaciones sociales de la delincuencia. Universitas Psychologica, 17(2), 1-14. https://doi.org/10.11144/Javeriana.upsy $17-2$. isrs

\section{RESUMEN}

El presente trabajo analiza las dinámicas de las representaciones sociales de la delincuencia en jóvenes universitarios de Venezuela $(n=305)$ y el País Vasco $(n=237)$ y la centralidad de la inseguridad subjetiva en sus principios organizadores. Siguiendo la metodología de Doise, Clèmence y Lorenzi-Cioldi (1993), se estudian los significados compartidos y diferenciales de la delincuencia, así como su anclaje en la percepción subjetiva del riesgo, experiencia de victimización y pertenencia a dos realidades nacionales. Los resultados muestran una representación más estereotipada de la delincuencia en los universitarios vascos asociando ciertos delitos a grupos y contextos criminógenos. Sin embargo, los venezolanos enfatizan las causas y consecuencias destructivas para la vida social y un estado de miedo e inseguridad, debido a una experiencia más cercana al delito. La percepción subjetiva del riesgo y la experiencia de victimización quedan asociadas a dichas representaciones, lo que permite la propuesta de nuevas prioridades y desafíos en los métodos utilizados para estudiar y combatir el miedo e inseguridad subjetiva.

\section{Palabras clave}

miedo al delito; inseguridad; victimización; representaciones sociales; delincuencia.

\begin{abstract}
This work analyses the dynamics of social representations with a sample of university students in Spain $(n=237)$ and Venezuela $(n=305)$ along with the centrality of subjective insecurity on their organizational principles. Following the methodology proposed by Doise, Clèmence, and Lorenzi-Cioldi (1993), the shared and differential meanings of delinquency are studied, as well as their anchoring in the subjective perception of risk, victimization experience and belonging to two national realities. Results show that Basque students represent delinquency stereotypically, associating some crime types to certain groups and criminogenic contexts related to young people, drugs, and immigration. Nevertheless Venezuelan students emphasise the causes of crime and its destructive consequences for social life, and a state of fear and insecurity due to a closer experience of crime. The subjective perception of risk and the victimization experiences are associated with these representations,
\end{abstract}


allowing us to propose new priorities and challenges in the methods used for studying and facing fear and subjective insecurity.

Keywords

fear of crime; insecurity; victimization; social representations; delinquency.

El delito tiene un importante impacto en las comunidades y en el bienestar personal. Este impacto no es sólo directo, vía victimización; la percepción subjetiva del riesgo y las respuestas emocionales ante la amenaza que supone la agresión alcanzan a muchos más ciudadanos y ciudadanas que el delito mismo. La literatura científica viene abordando este fenómeno a través del constructo denominado "miedo al delito", encontrando que las tasas objetivas de delito no siempre concuerdan con la percepción de la ciudadanía. A menudo, colectivos menos victimizados, según los datos oficiales de delincuencia, expresan más temor, lo que se ha venido denominando la "paradoja del miedo al delito" (Farrall, Jackson, \& Gray, 2008; Fattah, 1993); también la "paradoja espacial” (Vozmediano \& San-Juan, 2006) o la "paradoja invertida", en la que la inseguridad objetiva supera al miedo (Liebnitzky \& Montero, 2013). En la literatura científica, se han abordado a menudo las diferencias entre hombres y mujeres a este respecto (Ávila, Martínez-Ferrer, Vera, Bahena, \& Musitu, 2015; Robles, 2014), así como las diferencias en relación con la edad, e incluso se estudia el miedo al delito altruista, es decir, el que sentimos por la seguridad de otras personas significativas (Vozmediano, San-Juan, Vergara, \& Alonso-Alberca, 2017).

La investigación criminológica ha revelado de manera consistente la relevancia del contexto comunitario para comprender el temor ante el delito, analizando desde una perspectiva ecológica (Fernández-Ramírez, 2008; Van Beek, 2004) la influencia de las dinámicas psicosociales del área residencial en el nivel de inseguridad percibido. Las redes sociales, la satisfacción con el vecindario y la eficacia colectiva o la cohesión social reducirían el miedo al delito (Abdullah, Hedayati Marzbali, Maghsoodi
Tilaki, \& Bahauddin, 2015; Brunton-Smith, Jackson, \& Sutherland, 2014; Ferguson \& Mindel, 2007). La misma relación se encuentra con la cultura ciudadana y la satisfacción con la policía (Ruiz, 2007). Sin embargo, otras dinámicas incrementarían el miedo, por ejemplo, cuando la diversidad cultural y racial se interpreta como amenaza (Eitle \& Taylor, 2008). Si analizamos el temor como reacción ante una percepción de un peligro delictivo actual, en un lugar concreto, el diseño de los espacios urbanos y su cuidado y mantenimiento por parte de la comunidad, asociados a las nociones de incivilidad o desorden, toman a su vez relevancia (Doran \& Lees, 2005), poniendo de manifiesto el aspecto contextual (Jasso, 2013). Encontramos así un área en la literatura en la que progresivamente se van identificando los aspectos concretos del diseño urbano que impactan en el miedo al delito (p. ej., Foster, Knuiman, Wood, \& Giles-Corti, 2013; Hedayati Marzbali, Abdullah, \& Maghsoodi, 2016; Lorenc et al., 2013). Junto al contexto sociocultural y el situacional, los factores individuales constituyen un tercer elemento reconocido por la investigación como relevante en la comprensión del miedo al delito (San-Juan, Vozmediano, \& Vergara, 2012; Soomeren et al., 2008).

Por lo tanto, este juicio o interpretación subjetiva de vulnerabilidad no se realiza en un vacío social (Ferraro, 1995), sino que las características personales, relacionales y ecológicas contextualizan el proceso de interpretación de los riesgos potenciales y experiencias de victimización. Precisamente, las situaciones de victimización tanto de manera directa como indirecta, a través de la victimización de un familiar o amigo, incrementan el temor a los delitos (Baker, Nienstedt, Everett, \& McCleary, 1983). Sin embargo, en ausencia de experiencias personales, la fuente esencial de información son las noticias de los medios o incluso la rumorología sobre victimizaciones (Soto, 2005). De modo que resulta ineludible mencionar el papel de los medios de comunicación en la configuración de la percepción de inseguridad, con un 
tratamiento del delito a menudo sensacionalista; aunque el peso explicativo se comparte con las variables individuales, situacionales y ecológicas previamente mencionadas (Dowler, 2003; Molina-Jácome, 2014; Rodríguez \& Quinde, 2016; Scherman \& Etchegaray, 2013). Además, las percepciones e interpretaciones de los sujetos ante la noticia son clave: si la persona se identifica con la víctima o percibe que su contexto inmediato se parece al descrito, la imagen del riesgo puede asumirse y personalizarse (Farrall et al., 2008).

De todo ello se deduce que se trata de un fenómeno complejo, con raíces en distintos niveles, y que, al ser estudiado ampliamente por las ciencias sociales en las últimas décadas, se ha conceptualizado y definido de múltiples maneras. A menudo, se ha definido el miedo al delito como una respuesta emocional caracterizada por un sentimiento de ansiedad y peligro (p. ej., Ferraro, 1995). Los trabajos más recientes conciben el miedo al delito como la combinación de una estimación del riesgo de victimización, de naturaleza cognitiva, con una respuesta emocional que deriva, al menos en parte, de la anterior (Foster et al., 2013; Lorenc et al., 2012). Otros autores abogan por un constructo más inclusivo: la amenaza de victimización (Carro, Valera, \& Vidal, 2010; Valera \& Guàrdia, 2014) que contempla aspectos cognitivos, afectivos y conativos. Este último aspecto, la reacción conductual, puede implicar medidas de autoprotección, como la restricción de la libre movilidad en el espacio público urbano o incluso manifestaciones de xenofobia y miedo al extraño (Fernández-Ramirez, 2008; Lorenc et al., 2013; San-Juan et al., 2012), cuya relevancia pone a prueba la ambivalencia y el conflicto sociocognitivo respecto a la delincuencia y su afrontamiento psicosocial.

Junto a la heterogeneidad en las definiciones, encontramos además una diversidad de medidas que ponen de manifiesto que las cuestiones de operacionalización son cruciales (Vozmediano, San-Juan, \& Vergara, 2008). Las alternativas de medida más comunes durante décadas (las incluidas en encuestas de victimización gubernamentales) suscitan dudas en el campo metodológico, incluyendo riesgos de sobreestimación del miedo (Gray, Jackson, \& Farrall, 2008). Así, en los últimos años algunos autores exploran nuevas vías para la comprensión científica del miedo al delito, desde un punto de vista crítico con la literatura previa (Farrall \& Lee, 2009). Farrall, Jackson y Gray (2009) proponen distinguir entre el miedo experiencial, que corresponde a los episodios concretos de miedo que experimenta una persona y que varía según el tiempo, la localización y la situación; y, el miedo expresivo, aquel que se relata o expresa para compartir actitudes e inquietudes sobre los cambios sociales y el delito. Desde este último punto de vista, también se ha propuesto abordar los aspectos representacionales, culturales y comunitarios de las reacciones ante el delito, de un modo más amplio y menos reduccionista, a través de la denominación "(in)seguridad subjetiva" (SanJuan et al., 2012). Se trata de un concepto más amplio que el miedo al delito, aunque lo incluya, que trata de abarcar la riqueza y diversidad de las percepciones, emociones y reacciones ante el delito. El adoptar como referente teórico las representaciones sociales (Moscovici, 1976) en el estudio de la delincuencia, obedece a sus propias características, definidas por ser una forma social de conocimiento práctico, orientado hacia la comprensión y el dominio del entorno social y material y centrada fundamentalmente en la interpretación de los fenómenos sociales. Desde la perspectiva de Doise, Clèmence y Lorenzi-Cioldi (1993), las representaciones sociales serían aquellos principios organizadores de las relaciones simbólicas entre los individuos y grupos; marcos comunes de referencia que necesitan para relacionarse, que son generados a través de sistemas de intercambio y comunicación diversos y que a menudo se utilizan para justificar determinadas prácticas sociales. La delincuencia como problema social necesita ser afrontada tanto simbólica como materialmente (Wagner, Elejabarrieta \&Valencia, 1994), siendo dos los procesos que intervienen. Mediante la objetivación se transforman los vínculos abstractos de ideas pasadas, en un contenido mental concreto. El anclaje, por su parte, es 
el proceso por el cual los objetos no familiares son comparados e interpretados a la luz de fenómenos ya comprendidos. Esta perspectiva queda asociada a los estudios sobre miedo al delito a través del ya citado miedo expresivo, que trasciende la experiencia de temor personal ante una situación concreta, y se enmarca en lo que podríamos denominar percepciones subjetivas de la delincuencia, objeto de este trabajo.

El objetivo de esta investigación ha sido analizar las dinámicas de las representaciones sociales de la delincuencia y la relevancia de la inseguridad subjetiva en sus principios organizadores. Asimismo, se ha querido examinar el efecto de la diferenciación social vinculada al posicionamiento social del país, experiencias de victimización y percepción subjetiva del riesgo como realidades sociales y psicológicas, para explicar un problema sociopolítico como la delincuencia. El estudio de Gaffié (2006) reveló seis factores explicativos de la delincuencia, con representaciones ancladas principalmente en un enfoque psicológico e individualista, que impone cierto grado de responsabilidad al delincuente y la actuación sobre el comportamiento de los individuos (delincuentes, padres, educadores, etc.); la delincuencia asociada a causas sociales externas y factores que asocian la delincuencia a la personalidad de los actores. Estas dimensiones, similares a las encontradas en otros estudios previos (p. ej., Doise \& Papastamou, 1987; Furnham \& Henderson, 1983), parecen ser principios generales que generan y organizan el posicionamiento de los sujetos pertenecientes a diferentes categorías.

Sin embargo, no podemos obviar aquí que los aspectos culturales y ecológicos, así como la realidad delictiva de cada uno de los contextos, son diferentes. Concretamente, respecto al nivel de violencia, ésta es mucho más común en Latinoamérica que en los países de Europa Occidental. Las muertes por violencia son un $450 \%$ superiores (Soares \& Naritome, 2010). A nivel de país, las estadísticas internaciones sobre homicidios, realizada por la Oficina de las Naciones Unidas sobre Drogas y Delito, muestran que en Venezuela las tasas de homicidio han ascendido desde el año 2003 hasta la actualidad, siendo en el año 2015 de 57 por cada 100000 habitantes; mientras que en España se mantienen estables: se comienza dicho periodo con una tasa alrededor de 1 por cada 100000 habitantes, que desciende hasta 0.66 en la actualidad (United Nations Office on Drugs and Crime [UNODC], 2017). Pero, además, el País Vasco es una comunidad autónoma segura en comparación con el total del Estado.

¿Por qué y en qué condiciones las personas de diversos contextos sociopolíticos tienden a ofrecer diferentes explicaciones de un problema social? La hipótesis de partida es que si dos grupos tienen diferentes representaciones de un objeto (en este caso la delincuencia), la divergencia se fortalecerá en función de la diferenciación intergrupal (aquí el posicionamiento sociocultural). A la luz de la literatura revisada, en ambos contextos las representaciones y emociones evocadas estarán asociadas en parte con la experiencia de victimización, con la agenda de los medios de comunicación y serán coherentes con la vulnerabilidad y los procesos comunitarios y ecológicos asociados a cada país. La intensidad y frecuencia de los temores, e incluso la relación entre los conceptos mencionados, será probablemente distinta en ambos países, lo que puede contribuir a enriquecer la comprensión de las raíces de los miedos ante el delito, siguiendo la recomendación de Hirtenlehner y Farrall (2013) de abordar estudios comparativos entre países con contextos socioculturales, políticos e institucionales diversos.

Los resultados del estudio transcultural de Doise, Spini y Clèmence (1999) sobre la Declaración Universal de los Derechos Humanos, con estudiantes universitarios de 35 países de los cinco continentes, mostraron que en cada grupo nacional, las personas encuestadas tuvieron una mayor adhesión a los derechos básicos y sociales que al resto de los derechos, apoyando claramente la idea de una organización común de respuestas en varios países con áreas sociogeográficas diferentes, aunque los derechos colectivos se interpretaran de manera más diversa. Del mismo modo, los resultados de la investigación de Gaffié (2006) sobre las representaciones sociales de la delincuencia 
revelaron principios organizadores comunes, pero diversos posicionamientos políticos. Tal y como señala Harré (2012), en su estudio sobre la teoría del posicionamiento y las dimensiones morales de la psicología sociocultural, una diferencia fundamental entre las culturas es la concepción sobre el sistema de derechos y deberes. Los estudios realizados desde esta perspectiva (teoría del posicionamiento) incluyen desde reflexiones morales privadas de los grupos en interacción hasta los discursos y posicionamientos de los protagonistas de naciones o Estados.

La metodología del estudio es la propuesta por Doise et al. (1993), utilizada en otras investigaciones sobre los derechos humanos (Doise et al., 1999), con desarrollos más recientes en el ámbito sociopolítico (p. ej., Arnoso, Arnoso, \& Pérez-Sales, 2012; Cárdenas \& Blanco, 2004; Elcheroth, Doise, \& Reicher, 2011). Desde el citado modelo, en primer lugar, se contrasta el carácter compartido y estructurado de las representaciones sociales, haciendo referencia al proceso de objetivación (Moscovici, 1976). Posteriormente, se analizan los principios organizadores de las representaciones sociales, la heterogeneidad individual del posicionamiento dentro de la estructura representacional. Finalmente, se estudia el proceso de anclaje o diferenciación social, en el sentido de que las variaciones sistemáticas en los posicionamientos de los individuos se anclan en diferentes realidades o inserciones sociales (países) y experiencias psicológicas (percepción subjetiva del riesgo y experiencia de victimización).

\section{Método}

\section{Participantes}

La selección de la muestra se realizó a través de un muestreo no aleatorio. Participaron 542 estudiantes universitarios procedentes de Venezuela y el País Vasco. El sexo y la edad de los participantes fueron similares en ambos grupos. El $26.3 \%$ de la muestra estuvo constituido por hombres y el $73.7 \%$ por mujeres, con una media de edad de 22.39 años $(D E=5.7)$. Los sujetos se encontraban matriculados en primer y segundo curso de diferentes titulaciones de la Universidad Central de Venezuela $(n=305 ; 56.3 \%)$ y la Universidad del País Vasco $(n=237 ; 43.7$ \%).

\section{Variables e instrumento}

El instrumento está conformado por dos partes. La primera consta de un apartado de Asociación Libre de Palabras, en el que se les pide a los sujetos que escriban las primeras cuatro palabras que les vengan a la mente ante el estímulo "delincuencia". En la segunda, además de las variables principales que discriminan a la población según datos sociales básicos (sexo, edad, país, etc.), se toman en consideración diversos indicadores de victimización:

\section{Experiencia directa o indirecta de victimización}

Por un lado, se recoge información sobre la experiencia de victimización en un formato de respuesta Sí - No (p. ej., "iUd. o alguien cercano ha sido víctima de algún delito?"). Por otro lado, la cercanía o nivel de vinculación con la víctima en una escala tipo Likert de 4 puntos $(1=$ No conozco; 2 = Persona conocida $; 3=$ Familiar o amigo/a; 4 = Yo mismo/a) (p. ej., "¿Qué vinculación tiene con él/ella?").

\section{Percepción subjetiva del riesgo}

Se pregunta a los sujetos por la probabilidad subjetiva de victimización, en una escala tipo Likert con 3 opciones de respuesta ( $1=\mathrm{Nada}$; $3=$ Mucha) (p. ej., "iEn qué medida considera Ud. que tendría posibilidades de ser víctima de un delito?").

\section{Procedimiento}

Se diseñaron dos redacciones del instrumento adaptados a Venezuela y España, contando con la colaboración de colegas venezolanos en el proceso de traducción de doble ciego. 
Todos los sujetos contestaron el cuestionario de forma anónima y en situación colectiva, en el aula, durante el tiempo de una clase lectiva normal, después de solicitar los permisos correspondientes y contar con el consentimiento informado de los participantes.

\section{Análisis de datos}

Con los datos textuales, se realizó un análisis de contenido, utilizando el programa Systéme Portable pour l'Analyse des Données Textuelles ([SPAD-T]; Lebart \& Salem, 1994), para conocer el campo semántico de las representaciones sociales de la delincuencia, generándose categorías a partir de aquellas palabras de mayor frecuencia de aparición. En cada categoría, se reunieron las diferentes formas de una misma palabra con su expresión más común y se agruparon todas aquellas que poseían un evidente sentido en una categoría capaz de unificarlas (Di Giacomo, 1980), creando así un diccionario de palabras. El sistema de categorización seleccionado fue probado con tres jueces "ciegos" e independientes, con un grado de coincidencia interjueces (Indice Kappa 0.8-0.9), que permitió trabajar con un sistema de categorías altamente fiables. Para elaborar la matriz general de datos, se consideró la presencia (valor de uno, 1) o la ausencia (valor de cero, 0 ) de términos del diccionario al estímulo delincuencia.

Siguiendo el modelo trifásico (Doise et al., 1993), se analizaron, a través del Análisis Factorial de Correspondencias (AFC), las concepciones compartidas de la delincuencia y la centralidad del miedo y la inseguridad subjetiva en dichas representaciones. Posteriormente, para evidenciar los principios organizativos del campo representacional, se obtuvieron los posicionamientos individuales, por medio del AFC y el Análisis de Clasificación Jerárquica de los sujetos. Por último, se estudiaron los elementos de anclaje de los sistemas de creencias en función del país de pertenencia, la experiencia de victimización y la percepción subjetiva del riesgo, mediante el AFC de las palabras estímulo y el programa Posit del mismo.

\section{Resultados}

\section{Delincuencia y objetivación}

El análisis de contenido de las palabras que el alumnado universitario asoció al concepto de "delincuencia" se realizó tomando aquellas que tuvieron una frecuencia superior a tres. El diccionario de palabras fue creado a partir del total de las asociaciones al estímulo delincuencia. La producción total de palabras se redujo a dimensiones con el mismo significado. Así, de un total de 2082 palabras, se extrajeron 75 categorías que equivalen a un $96.69 \%$ del total de los conceptos emitidos.

De acuerdo a los resultados recogidos en la Tabla 1, y utilizando el criterio de atributos prototípicos (aquellos que han sido nombrados alrededor del $10 \%)$, los elementos clave que conforman las representaciones sociales de la delincuencia se refieren fundamentalmente al robo, la pobreza, los delitos, la violencia, la marginación, el asesinato, la necesidad, las drogas, los jóvenes, la prisión, la falta de educación y la inseguridad.

Tabla 1

Modalidades activas y frecuencias asociadas a la delincuencia

\begin{tabular}{lrllll}
\hline \multicolumn{1}{c}{$\begin{array}{c}\text { Modalidades } \\
\text { activas }\end{array}$} & $n$ & $\begin{array}{c}\text { Modalidades } \\
\text { activas }\end{array}$ & $n$ & Modalidades activas & $n$ \\
\hline Robo & 174 Sociedad & 26 Inmigración & 9 \\
Pobreza & 110 Delincuentes & 26 Resentimiento & 9 \\
Delito & 95 Actual & 24 Policía & 9 \\
Violencia & 92 Ladrones & 24 Víctima & 9 \\
Marginación & 82 Abuso & 20 Abandono & 9 \\
Asesinato & 81 Hambre & 19 Hombres & 9 \\
Necesidad & 78 Atraco & 19 Terrorismo & 8 \\
Drogas & 75 Desempleo & 18 Enfermedad mental & 8 \\
Jóvenes & 63 Drogadicción & 18 Personas & 8 \\
Prisión & 54 Agresión & 18 Angustia & 8 \\
Falta de educación & 49 Castigo & 17 Odio & 8 \\
Inseguridad & 48 Desviación social & 17 Desesperación & 7 \\
Socio estructurales & 43 Soluciones & 15 Dinero & 7 \\
Peligro & 42 Falta valores & 15 Rabia & 7 \\
Muerte & 42 Sufrimiento & 14 Control & 6 \\
Maldad & 41 Corrupción & 14 Ley & 6 \\
Armas & 36 Asalto & 14 Enfermedades & 5 \\
Problema & 34 Malandros & 13 Negativo & 5 \\
Miedo & 31 Exclusión & 13 Inconsciencia & 5 \\
Contexto & 30 Maltrato & 12 Inmoral & 5 \\
Falta de familia & 29 Desorden & 11 Pena & 5 \\
Daño & 28 Vagancia & 10 Ayuda & 5 \\
Violación & 27 Sistema político & 10 Justicia & 4 \\
Desadaptación & 27 Educación & 10 Mafias & 4 \\
Antisociales & 26 Injusticia & 10 Problemas psicológicos & 4 \\
\hline
\end{tabular}




\section{Principios organizadores de las representaciones sociales de la delincuencia}

Con el fin de indagar en la articulación de los sistemas de creencias, se realizó un AFC utilizando como modalidades activas las palabras asociadas al estímulo "delincuencia". Estas dan cuenta de la objetivación: la forma como un fenómeno más o menos complejo se transforma en metáforas, imágenes y conceptos concretos. El análisis produjo 15 factores cuyo valor propio medio $(S \mathrm{vp}=100 / 15)$ fue de 4.5, por encima de dicho valor se encontraban los cinco primeros factores, los cuales presentaban una traza de la inercia acumulada del 12.3 \%. La extracción final de dichos factores se realizó sobre aquellas dimensiones con valor propio superior a $0.54 \quad(4.39 \%$ de la inercia), correspondiendo a un total de dos que mostraron una traza de la inercia acumulada del $5.39 \%$ (2.83 \% y $2.56 \%$, respectivamente), adecuada en este tipo de AFC. Un número mayor habría hecho extremadamente dificultosa la interpretación. Posteriormente, el análisis produjo la tabla de coordenadas, contribuciones y cosenos cuadrados. Para la interpretación de los ejes factoriales, se necesitan tanto las coordenadas como las contribuciones de cada modalidad a la composición del factor y las contribuciones relativas o cosenos cuadrados que constituyen el porcentaje explicado por el factor. Esto puede evitar interpretaciones erróneas basadas simplemente en los gráficos de las proyecciones de las modalidades en los ejes factoriales. Así, el criterio utilizado para especificar las mayores contribuciones, es que fuera superior a la media (en nuestro caso 100/75 modalidades $=1.33$ ).

De las 75 modalidades encontradas, son 23 las que presentan una contribución superior a la media (1.33) en el primer factor y una contribución relativa del factor a la modalidad que oscila entre el $3 \%$ y el $24 \%$. Este primer factor se encuentra definido en su polo negativo por 12 modalidades con una contribución superior a la media: falta de familia (7.5), falta de educación (6.6), necesidad (6.5), abandono (5.1), sociedad (4.9), pobreza (4), falta de valores (3.6), hambre (2.6), ayuda (2.6), inconsciencia (1.9), desempleo (1.6) y soluciones (1.6). Sin embargo, su polo positivo se encuentra definido por 11 modalidades: robo (8.2), asesinato (5.4), delito (4), prisión (2.6), delincuentes (2.4), violación (2.3), armas (2), agresión (1.6), violencia (1.4), atraco (1.4) y asalto (1.3). De esta manera, se puede decir que este primer eje factorial opone una explicación de la delincuencia basada en las causas externas, en su polo negativo, a una visión asociada a los delitos amenazantes ante la integridad personal y la prisión como respuesta, en su polo positivo.

El segundo factor está compuesto por 24 modalidades que presentan una contribución superior a la media (1.33) y una contribución relativa del factor a la modalidad que oscila entre el $3 \%$ y el $16 \%$. Este segundo eje, en su polo negativo se encuentra definido por palabras como maldad (8.7), delito (8.4), daño (8.4), miedo (4.6), sufrimiento (4.5), angustia (4), problema (3.9), injusticia (3.3), actual (3.2), desviación social (2.7), negativo (2.5), desorden (2.3), control (2.2), enfermedades (1.7) e inseguridad (1.5). En su polo positivo, en cambio, queda definido por modalidades referidas a las drogas (4.8), robo (3.3), asesinato (2.8), contexto (2.8), jóvenes (2.3), violación (1.9), inmigración (1.8), prisión (1.8) y armas (1.6). Se observa, por tanto, que este segundo eje factorial opone una visión de la delincuencia que hace referencia a las amenazas y contextos criminógenos, en su polo positivo, a una visión en la que las respuestas emocionales y el impacto en la comunidad se hacen manifiestos con una evaluación moral, en su polo negativo.

\section{Posicionamientos individuales}

Con el fin de analizar la segunda hipótesis del Modelo Trifásico de Doise et al. (1993), se realizó un Análisis de Clasificación Jerárquica de los sujetos, cuyos centros fueron proyectados en el cruce de los dos primeros factores del AFC (Figura 1). 


\section{Figura 1}

Representación gráfica de los dos primeros factores del AFC

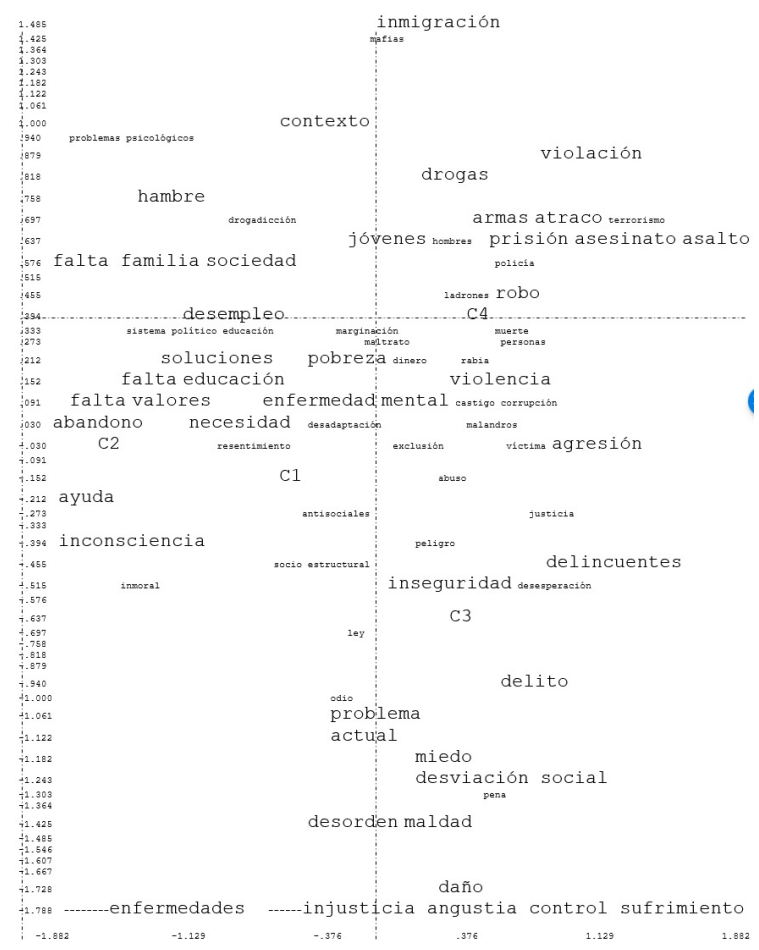

El análisis produjo cuatro agrupaciones claras de sujetos: 1) Una primera asociada a Percepciones compresivas del delito, en la medida en que se refiere al origen de la delincuencia, cercana a las palabras como pobreza, falta de educación, falta de familia, necesidad, marginación, actual, problemas socioestructurales, falta de valores, sociedad y drogadicción; 2) Una segunda, que se podría denominar Percepciones ambivalentes por la referencia a cuestiones tales como abandono, inconsciencia, ayuda, necesidad y odio; 3) Una tercera agrupación que se denominaría Percepciones normativas puesto que se refiere a los delitos, delincuentes, desviación social, ley, víctima, pena, negativo, antisocial y justicia y, 4) por último, una cuarta denominada Percepciones de amenaza, puesto que se encuentra cercana a las palabras robo, asesinato, armas, muerte, violación, drogas, atraco, violencia, prisión y agresión. El primer clúster reagrupó 221 sujetos, el segundo 16 , el tercero 84 y el cuarto 221 sujetos.

\section{Posicionamientos en función del país, experiencia de victimización y percepción subjetiva del riesgo}

A continuación, se exponen los posicionamientos de las pertenencias al país, experiencia de victimización y percepción subjetiva del riesgo como modalidades suplementarias. En el AFC, los índices que informan sobre la relación entre el eje factorial y la modalidad suplementaria son las coordenadas en el eje y el valor prueba, que constituyen una constatación de significatividad sobre el posicionamiento de una modalidad en un eje. Se considera significativa la modalidad donde los valores test son superiores a 2 en valor absoluto, los cuales corresponden a un nivel de significatividad aproximado de 0.95 .

En relación con la pertenencia al país, se constata que ambas realidades socioculturales se encuentran relacionadas significativamente con el primer eje factorial. Así, éste se halla positivamente relacionado con la pertenencia al País Vasco (7.1) y negativamente con la pertenencia a Venezuela (-7.1). Recordemos que el primer factor oponía una visión de la delincuencia referida a las causas externas de la delincuencia (en el polo negativo) a representaciones basadas en los tipos de delito amenazantes para la integridad personal y la prisión como respuesta (en el polo positivo). La experiencia directa $(-6.1)$ e indirecta $(-3.7)$ de victimización se encuentra negativamente relacionada con este factor, mientras que el no haber tenido ninguna experiencia de victimización (5.1) se relaciona positivamente. En relación con la percepción subjetiva del riesgo, este primer factor opone a los sujetos que perciben una alta probabilidad de ser víctima (relacionados negativamente, -3.6), frente a los sujetos que perciben baja probabilidad (relacionados positivamente, 4).

En relación con el segundo factor, que opone una visión cognitiva de la delincuencia que enfatiza la percepción de amenaza y contextos criminógenos (polo positivo) a sistemas de creencias centrados en las respuestas emocionales e impacto en la comunidad (polo 
negativo), las dos modalidades de pertenencia al país se relacionan significativamente. Los estudiantes venezolanos (-5.6) se hallan relacionados negativamente, mientras que los vascos (5.6) lo hacen positivamente. En relación con la experiencia de victimización, el hecho de no conocer a nadie que haya sido víctima (5.5) y la experiencia directa de victimización (2.2), se relaciona positivamente, mientras que la experiencia indirecta a través de algún familiar o amigo que haya sido víctima $(-7.0)$ se encuentra negativamente relacionada. La percepción subjetiva del riesgo (-3.6) se relaciona negativamente con este segundo factor y la percepción de riesgo moderado (3.5), positivamente.

\section{Discusión}

En el presente estudio se han analizado las dinámicas de las representaciones sociales de la delincuencia en jóvenes universitarios venezolanos y vascos y la centralidad de la inseguridad subjetiva en sus principios organizadores. Asimismo, se ha querido examinar el efecto de la diferenciación vinculada al posicionamiento social del país, experiencia de victimización y percepción subjetiva del riesgo (como inserciones sociales y psicológicas). Las creencias compartidas ponen de manifiesto la relevancia de la inseguridad como reacción emocional entre los participantes, además de la ecología de la actividad delictiva asociada a contextos de pobreza, necesidad, marginación, drogas, así como la referencia al robo y delitos que implican acometimiento personal. Estas representaciones se modulan en torno a dos dimensiones de significado. La primera de ellas opone una explicación de la delincuencia basada en causas externas como la pobreza, necesidad o dificultades de socialización, a otra visión que hace referencias explícitas a los tipos de delito más amenazantes para la integridad personal y a la prisión como respuesta. En la segunda dimensión, destaca la centralidad de las nociones de desviación social y desorden (Doran \& Lees, 2005), sus consecuencias destructivas en la vida social y comunitaria y un conjunto de imágenes centradas en un estado emocional de miedo e inseguridad. Estas representaciones contrastan con una visión estereotipada centrada en las referencias explícitas a los tipos de delito y delincuentes que incorpora la relevancia del contexto y la diversidad cultural y/o racial (Eitle \& Taylor, 2008). Estas dimensiones, en parte coincidentes con las del estudio de Gaffié (2006), constituyen los principios que generan y organizan el posicionamiento de los sujetos pertenecientes a diversas categorías sociales.

El estudio de la dinámica representacional a través de los niveles de análisis (Doise et al., 1993) muestra la compleja articulación de los elementos de objetivación y anclaje, permitiendo ahondar en las diferencias que se esconden detrás de los aspectos consensuados. Se identifican diferencias en función del país o contexto sociocultural, la percepción del riesgo y la experiencia de victimización, permitiendo contextualizar los resultados de la investigación en miedo al delito que han optado por otras metodologías. Con respecto al país, el estudio del posicionamiento revelado por los polos de las dos dimensiones resalta, por un lado, la persistente saliencia de las apuestas tradicionales de la prisión como respuesta ante los delitos amenazantes ante la integridad personal entre los estudiantes vascos, asociado a explicaciones individualistas consistentes con la ideología que prevalece en las sociedades occidentales (Dubois, 2003), que se oponen a una concepción de la delincuencia basada en causas externas entre los venezolanos. Además, controversias contemporáneas como las relacionadas con la inmigración se reflejan probablemente en oposiciones entre las evocaciones de naturaleza más cognitiva vinculadas a la amenaza que suponen las formas de victimización de diversa gravedad y su asociación con ciertos contextos marginales y grupos de población (jóvenes e inmigrantes) más salientes entre los estudiantes vascos, y las consecuencias destructivas para la vida social y comunitaria con diversas reacciones emocionales de sufrimiento, miedo e inseguridad, entre los venezolanos, debido probablemente a una experiencia más cercana al delito. En 
términos generales, los resultados en relación con el país parecen coherentes con lo que sabemos de contexto sociopolítico y clima emocional. El posicionamiento asociado a la percepción subjetiva del riesgo y la experiencia directa e indirecta de victimización van en la misma dirección. Como se ha puesto de manifiesto durante décadas en la literatura, desde la definición de la citada "paradoja del miedo al delito" (Fattah, 1993), la relación entre las mediciones objetivas del riesgo de victimización con la percepción subjetiva de riesgo y con el propio miedo distan de ser lineales y sencillas. Además, para la mayoría de personas que no tienen experiencia directa de victimización, como es el caso mayoritariamente de los estudiantes vascos, los medios de comunicación o la información de su red de relaciones serán sus fuentes de información sobre el delito (Soto, 2005) y uno de los factores que determinarán estas representaciones vinculadas a la amenaza y los contextos criminógenos. Por lo tanto, las representaciones sociales de la delincuencia lejos de estar confinadas en opiniones homogéneas y consensuadas, como ocurre con el propio miedo al delito, dejan espacio para una variabilidad interindividual más dependiente de factores socioculturales y situaciones individuales (Doise et al., 1993; Gaffié, 2006; San-Juan et al., 2012).

Reconociendo que el crimen causa daños a personas y comunidades y genera niveles de inseguridad y temor, a la luz de la literatura revisada se podría esperar que tanto la probabilidad subjetiva de ser víctima como la propia experiencia de victimización se asociara con actitudes más punitivas que culpabilizan al delincuente. Sin embargo, los hallazgos encontrados en este estudio sugieren que una mayor experiencia con el delito conlleva una actitud más comprensiva que incorpora los condicionantes de vida, lejos de posturas punitivas inducidas por el discurso político y mediático. Estas actitudes pueden constituir un contexto favorable a la movilización del capital social para la reducción del miedo (Ferguson \& Mindel, 2007), ya que trabajos recientes muestran que la eficacia colectiva, la construcción social de la comunidad y el apego emocional al barrio se asocian con un menor nivel de miedo (Williams, 2015). La visión comprensiva sería facilitadora para trabajar sobre estos aspectos, evitando conductas disfuncionales y de malestar social. En los contextos más seguros, puede ser un reto desmitificar las representaciones estereotipadas respecto de los contextos criminógenos, contribuyendo en la reducción de actitudes de intolerancia y temor hacia ciertos grupos.

Los resultados obtenidos sugieren líneas de discusión en relación con la aportación de la teoría de las representaciones sociales como herramienta teórico-práctica en el estudio del miedo al delito y la inseguridad subjetiva. Frente a la alternativa de incluir una escala concreta, la metodología propuesta ofrece elementos que permiten ampliar la comprensión de la parte expresiva del miedo al delito, en términos de Farrall et al. (2009). A pesar de la naturaleza descriptiva del estudio, el análisis de las frecuencias parece ya interesante. La clasificación muestra que las frecuencias más altas se refieren al robo y la pobreza, representadas en el $32 \%$ y $20 \%$ de los sujetos, respectivamente. La inseguridad y el miedo se hacen emergentes sin forzar a los sujetos a tomar una posición al respecto, asociándose asimismo con la desviación, el desorden y los temas ecológicos, al mismo tiempo que emergen otras reacciones emocionales como la ira, que aparece en este trabajo como sentimiento de indignación o injusticia. Todo ello enriquece el estudio del miedo al delito, reconociendo que la seguridad subjetiva es compleja y que el miedo no es la única emoción posible ante el delito, como ya señalaron Hartnagel y Templeton (2012).

Una de las limitaciones del trabajo estaría relacionada con la muestra de estudio. Para continuar profundizando en la comprensión de la inseguridad subjetiva y las representaciones sociales de la delincuencia, en futuras investigaciones sería conveniente contemplar otras variables que se vienen explorando en la literatura (género, edad, orientación política) y trabajar con otros grupos de edad, así como en contextos cultural y 
criminológicamente diversos. Es cierto que el trabajar con una muestra de conveniencia que no es representativa de la población impide hacer generalizaciones, sin embargo, sí permite extraer conclusiones para establecer líneas de trabajo futuro. Metodológicamente, sería deseable explorar la interacción de variables sociales y psicológicas como el país y la victimización y el peso relativo de cada una de ellas en la explicación de las representaciones sociales de la delincuencia. Asimismo, sería importante complementar la metodología propuesta con una de tipo mixto que incluya instrumentos de fiabilidad contrastada, de cara a una progresiva integración del conocimiento que se beneficie de las fortalezas de cada una de estas estrategias. Todo ello redundará en una mejor comprensión de las dinámicas del temor y las reacciones ciudadanas ante el delito, pudiendo además contribuir a plantear propuestas más ajustadas a las realidades sociales y psicológicas para la construcción colectiva de la seguridad objetiva y subjetiva, en distintos contextos.

\section{Referencias}

Abdullah, A., Hedayati Marzbali, M., Maghsoodi Tilaki, M. J., \& Bahauddin, A. (2015). Territorial features, disorder and fear of crime in residential neighbourhoods in Malaysia: Testing for multigroup invariance. Global Crime, 16(3), 197-218. http://dx.doi.org/10.1080/174405 72.2015.1019611

Arnoso, M., Arnoso, A., \& Pérez-Sales, P. (2012). Representaciones sociales del pasado: la dictadura militar argentina en la memoria colectiva. Revista de Psicología Social, 27(3), 259-272. http://dx.doi.org/10.1174/021347 412802845540

Ávila, M. E., Martínez-Ferrer, B., Vera, J. A., Bahena, A., \& Musitu, G. (2015). Victimización, miedo al delito y cambios en las rutinas cotidianas en un contexto de alta criminalidad, en función del género. Revista Española de Investigación Criminológica, 13,
1-22. Recuperado de https://dialnet.unirioj a.es/servlet/articulo?codigo $=5395416$

Baker, M., Nienstedt, B., Everett, R., \& McCleary, R. (1983). The impact of crime wave: Perceptions, fear and confidence in the police. Law $\mathcal{E}$ Society Review, 17(2), 319-336. http://dx.doi.org/10.2307/305335 0

Brunton-Smith, I., Jackson, J., \& Sutherland, A. (2014). Bridging structure and perception: On the neighbourhood ecology of beliefs and worries about violent crime. British Journal of Criminology, 54(4), 503-526. http ://dx.doi.org/10.1093/bjc/azu020

Cárdenas, M., \& Blanco, A. (2004). Las representaciones sociales del movimiento antiglobalización. Revista de Psicología Política, 28, 27-54. Recuperado de https://www.uv.es/garzon/psicologia\%20 politica/N28-2.pdf

Carro, D., Valera, S., \& Vidal, T. (2010). Perceived insecurity in the public space: Personal, social and environmental variables. Quality and Quantity, 44(2), 303-314. http://dx.doi.org/10.1007/s11135 $-008-9200-0$

Di Giacomo, J. P. (1980). Intergroup alliances and rejections within a protest movement (Analysis of the social representations). European Journal of Social Psychology, 10, 329-344. http://dx.doi.org/10.1002/ejsp.24 20100402

Doise, W., Clèmence, A., \& Lorenzi-Cioldi, F. (1993). The quantitative analysis of social representations. Londres: Harvester Wheatsheaf.

Doise, W., \& Papastamou, S. (1987). Représentation sociales des causes de la delinquance: croyances generales et cas concrets. Deviance et Societé, 11(2), 153-162. http://dx.doi.org/10.3917/ds.282. 0179

Doise, W., Spini, D., \& Clèmence, A. (1999). Human rights studied as social representations in a cross-national context. European Journal of Social Psychology, 29, 1-29. http://dx.doi.org/10.1002/ 
(SICI) 1099-0992(199902)29:1<1::AIDEJSP909>3.0.CO;2-\#

Doran, B. J., \& Lees, B. G. (2005). Investigating the spatiotemporal links between disorder, crime, and the fear of crime. Professional Geographer, 57, 1-12. http://dx.doi.org/10.1 $111 / j .0033-0124.2005 .00454 . x$

Dowler, K. (2003). Media consumption and public attitudes toward crime and justice: The relationship between fear of crime, punitive attitudes, and perceived police effectiveness. Journal of Criminal Justice and Popular Culture, 10(2), 109-126. Recuperado de https://www.albany.edu/scj/ jcjpc/vol10is2/dowler.html

Dubois, N. (2003). A sociocognitive approach to social norms. Londres: Routledge.

Eitle, D., \& Taylor, J. (2008). Are Hispanics the new 'threat'? Minority group threat and fear of crime in Miami-Dade County. Social Science Research, 37, 1102-1115. http://dx. doi.org/10.1016/j.ssresearch.2008.05.005

Elcheroth, G., Doise, W., \& Reicher, S. (2011). On the knowledge of politics and the politics of knowledge: How a social representations approach helps us rethink the subject of political psychology. Political Psychology, 32 (5), 729-758. http://dx.doi.or g/10.1111/j.1467-9221.2011.00834.x

Farrall, S., Jackson, J., \& Gray, E. (2008). La trascendencia cultural y social del miedo a la delincuencia. En A. Serrano Maíllo \& J. L. Guzmán Dálbora (Eds.), Procesos de infracción de normas y de reacción a la infracción de normas: dos tradiciones Criminológicas (pp. 233-276). Madrid: Dykinson.

Farrall, S., Jackson, J., \& Gray, E. (2009). Social order and the fear of crime in contemporary times. Oxford: Oxford University Press.

Farrall, S., \& Lee, M. (Eds.). (2009). Fear of crime: Critical voices in an age of anxiety. Abingdon, UK: Routledge-Cavendish.

Fattah, E. A. (1993). Research on fear of crime: Some common conceptual and measurement problems. En W. Bilsky, C. Pfeiffer \& P. Wetzels (Eds.), Fear of crime and criminal victimization (pp. 45-70). Sttutgart: Enke.

Ferguson, K. N., \& Mindel, C. H. (2007). Modeling fear of crime in Dallas neighborhoods: A test of social capital theory. Crime $\mathcal{E}$ Delinquency, 53, 322-349. http://dx.doi.org/10.1177/001112 8705285039

Fernández-Ramírez, B. (2008). Seis hipótesis de trabajo para entender la delincuencia y el miedo al delito. Revista Española de Investigación Criminológica, 6, 1-25. Recuperado de http://www.criminologia.ne t/pdf/reic/ano6-2008/a62008art6.pdf

Ferraro, K. F. (1995). Fear of crime: Interpreting victimization risk. Albany, NY: SUNY Press.

Foster, S., Knuiman, M., Wood, L., \& GilesCorti, B. (2013). Suburban neighbourhood design: Associations with fear of crime versus perceived crime risk. Journal of Environmental Psychology, 36, 112-117. http ://dx.doi.org/10.1016/j.jenvp.2013.07.015

Furnham, A., \& Henderson, M. (1983). Lay theories of delinquency. European Journal of Social Psychology, 13, 107-120. http://dx.doi .org/10.1002/ejsp.2420130202

Gaffié, B. (2006). Effect of political positioning on explanations of delinquency: An experimental study of social differentiation and representation. Political Psychology, 27(3), 403-421. http://dx.doi.org/10.1111/j $.1467-9221.2006 .00507 . x$

Gray, E., Jackson, J., \& Farrall, S. (2008). Reassessing the fear of crime. European Journal of Criminology, 5, 363-380. http://dx .doi.org/10.1177/1477370808090834

Harré, R. (2012). Positioning theory: Moral dimensions of social-cultural psychology. En J. Valsiner (Ed.), The Oxford handbook of culture and psychology (pp. 191-206). Nueva York: Oxford University Press. http://dx.doi.org/10.1093/oxfordhb/9 780195396430.013.0010

Hartnagel, T. F., \& Templeton, L. J. (2012). Emotions about crime and attitudes to punishment. Punishment $\mathcal{B}$ Society, 14(4), 452-474. http://dx.doi.org/10.1177/146247 4512452519 
Hedayati Marzbali, M., Abdullah, A., \& Maghsoodi Tilaki, M. J. (2016). The effectiveness of interventions in the built environment for improving health by addressing fear of crime. International Journal of Law, Crime and Justice, 45, 120-140. http://dx.doi.org/10.1016/j.ijlcj.2 015.12 .002

Hirtenlehner, H., \& Farrall, S. (2013). Anxieties about modernization, concerns about community, and fear of crime: Testing two related models. International Criminal Justice Review, 23, 5-24. http://dx.doi.org/10.1177/ 1057567712475307

Jasso, C. (2013). Percepción de inseguridad en México. Revista Mexicana de Opinión Pública, 15, 12-29. http://dx.doi.org/10.101 6/S1870-7300(13)72319-6

Lebart, L., \& Salem, A. (1994). Statistique textuelle. París: Dunod.

Liebnitzky, J., \& Montero, M. (2013). Miedo al crimen en estudiantes de la ciudad de Caracas. Psicologia E Sociedade, 25(1), 152-162. http://dx.doi.org/10.1590/S010271822013000100017

Lorenc, T., Clayton, S., Neary, D., Whitehead, M., Petticrew, M., Thomson, H., ... Renton, A. (2012). Crime, fear of crime, environment, and mental health and wellbeing: Mapping review of theories and causal pathways. Health and Place, 18(4), 757-765. http://dx.doi.org/10.1016/j.health place.2012.04.001

Lorenc, T., Petticrew, M., Whitehead, M., Neary, D., Clayton, S., Wright, K., ... Renton, A. (2013). Environmental interventions to reduce fear of crime: Systematic review of effectiveness. Systematic Reviews, 2, 30. htt p://dx.doi.org/10.1186/2046-4053-2-30

Molina-Jácome, I. (2014). Miedo al crimen y medios de comunicación: una revisión de la literatura. Revista Criminalidad, 56(3), 9-23. Recuperado de http://www.scielo.org.co/scielo.php?scri $\mathrm{pt}=$ sci_arttext $\&$ pid $=\mathrm{S} 1794-31082014000$ 300002

Moscovici, S. (1976). Social influence and social change. Londres: Academic.
Robles, A. L. (2014). Miedo en las calles: principal emoción de la inseguridad pública delictiva. Un estudio criminológico y de género. Revista del Instituto de Ciencias Jurídicas de Puebla, 8(34), 81-100. Recuperado de http://www.revistaius.com/i ndex.php/ius/article/view/123/514

Rodríguez, J. A., \& Quinde, M. (2016). Miedo al delito y medios de comunicación tradicionales. Espacio Abierto: Cuaderno Venezolazo de Sociología, 25(2), 145-166. Recuperado de https://www.researchgate.net/publicatio n/305386061_Miedo_al_delito_y_medios_ de_comunicacion_tradicionales

Ruiz, J. I. (2007). Procesos sociales relacionados con el miedo al crimen, la satisfacción con la policía y la victimizacion: el caso de la cultura ciudadana. International E-journal of Criminal Sciences, 1, 1-29. Recuperado de https://dialnet.unirioja.es/servlet/articul $\mathrm{o}$ ? codigo $=4876031$

San-Juan, C., Vozmediano, L., \& Vergara, A. (2012). Self-protective behaviours against crime in urban settings: An empirical approach to vulnerability and victimization models. European Journal of Criminology, 9(6), 652-667. http://dx.doi.org/10.1177/1 477370812454369

Scherman, A., \& Etchegaray, N. (2012). Consumo de noticias y temor al delito en Chile. Estudios sobre el Mensaje Periodístico, 19(1), 563-575. http://dx.doi.org/10.5209/r ev_ESMP.2013.v19.n1.42539

Soares, R. R., \& Naritomi, J. L. (2010). Understanding high crime rates in Latin America: The role of social and policy factors. En R. Di Tella, S. Edwards \& E. Schargrodsky (Eds.), The economics of crime: Lessons for and from Latin America (pp. 19-55). Chicago: University of Chicago Press.

Soomeren, P., Schillings, M., Smits, N., Woldendorp, T., Jongejan, A., Davey, C. ... Marselle, M. (2008). Reducing the fear factor. Guidance for addressing fear of crime and insecurity within urban development. Londres: Design Against Crime Solution 
Centre, University of Salford. Recuperado de http://www.securefit.org/downloads/files /Reducing\%20the\%20Fear\%20Factor.pdf

Soto, S. (2005). La influencia de los medios en la percepción social de la delincuencia. Revista Electrónica de Ciencia Penal y Criminología, 7, 1-46. Recuperado de http://criminet.ugr. es/recpc/07/recpc07-09.pdf

United Nations Office on Drugs and Crime. (2017). Homicide statistics. Recuperado de https://www.unodc.org/documents/gsh/ pdfs/GLOBAL_HOMICIDE_Report_ExS um spanish.pdf

Valera, S., \& Guàrdia, J. (2014). Perceived insecurity and fear of crime in a city with low-crime rates. Journal of Environmental Psychology, 38, 195-205. http://dx.doi.org/1 0.1016/j.jenvp.2014.02.002

Van Beek, G. (2004). Feeling (un)safe and (in) secure in the risk society. Leuven, BE: Katholieke Universiteit Leuven. Recuperado de http://statbel.fgov.be/studie s/ac084_en.pdf

Vozmediano, L., \& San-Juan, C. (2006). Empleo de sistemas de información geográfica en el estudio del miedo al delito. Revista Española de Investigación Criminológica, 2, 1-11.

Vozmediano, L., San-Juan, C., Vergara, A. I., \& Alonso-Alberca, N. (2017). "Watch out, sweetie": The impact of gender and offence type on parents' altruistic fear of crime. Sex Roles, 77(9-10), 676-686. http://dx.doi.org/ 10.1007/s11199-017-0758-7

Vozmediano, L., San-Juan, C., \& Vergara, A. (2008). Problemas de medición de miedo al delito: algunas respuestas teóricas y técnicas. Revista electrónica de Ciencia Penal y Criminología, 10, 1-15. Recuperado de http://criminet.ugr.es/recpc/10/recpc10. 07.pdf

Wagner, W., Elejabarrieta, F. \&Valencia, J. F. (1994). Estabilidad de las representaciones sociales de la guerra y la paz en dos países. Revista de Psicología Social y Personalidad, 10(2), 123-143.

Williams, S. A. (2015). Perceptions of the police and fear of crime: The role of neighborhood social capital (Tesis de maestría inédita). Graduate College of Bowling Green State University, Ohio. Recuperado de https://etd.ohiolink.edu/!etd.send file?a ccession $=$ bgsu $1440351447 \&$ disposition $=\mathrm{i}$ nline

\section{Notas}

* Artículo de investigación. Este trabajo ha sido realizado gracias a la financiación del Vicerrectorado de Investigación de la Universidad del País Vasco y la inestimable colaboración de la Universidad Central de Venezuela. 